\title{
APPLICATION OF DENDRIMERS AND QUANTUM DOTS IN CANCER DIAGNOSIS AND THERAPY
}

Emir Horozić, Sabina Begić, Amira Cipurković, Nusreta Suljkanović, Seida Mujanović

(C) by Acta Medica Saliniana ISSN 0350-364X

Type of manuscript: Professional papers

Title:

APPLICATION OF DENDRIMERS AND QUANTUM DOTS IN

CANCER DIAGNOSIS AND THERAPY

\section{Authors:}

Emir Horozić ${ }^{1}$, Sabina Begić ${ }^{1}$,

Amira Cipurković

Nusreta Suljkanović́ ${ }^{2}$ Seida Mujanović ${ }^{2}$

DOI: $10.5457 / 450$

\section{Afiliations:}

${ }^{1}$ Faculty of Technology, University of Tuzla, Univerzitetska 8, 75000 Tuzla, Bosnia and Herzegovina,

${ }^{2}$ Faculty of Natural Sciences, University of Tuzla, Univerzitetska 4, 75000 Tuzla, Bosnia and Herzegovina
The use of nanotechnology in the diagnosis of diseases, treatment and monitoring of cancer patients has become a subject of study for the last 20 years. This paper presents brief overview of general characteristics, synthesis and application of dendrimers and quantum dots (QDs) in oncology. Dendrimers and quantum dots (QDs) are nanostructures whose application in oncology is still being examined. QDs are used in magnetic resonance, wherein very well detect the location of tumor. Dendrimers are intensively tested, especially in cancer therapy, because their extremely small dimensions, they have the ability to pass through the mucous barriers and vascular pores, enabling safe delivery of the drug to the tumor cells.

Keywords: QDs, dendrimers, cancer, nanomedicine, drug delivery.

\section{INTRODUCTION}

The first appearance of the term "Nanotechnology" was 1974. in the conference paper, "On the basic concept of Nanotechnology" by Tokyo Science University Professor, Norio Taniguchi. [1] Nanotechnology is a science that deals with

nanometer-scale particles ie. nanoparticles, in the size range 1 to $100 \mathrm{~nm}$, to create functional devices, materials, and systems for numerous applications. Nanomaterials are categorised according to their dimensions as shown in Table 1. [2]

Table 1. Categorisation of nanomaterials according to their dimensions

\begin{tabular}{l|l}
\hline Nanomaterial dimension & Example \\
\hline All three dimensions $<100 \mathrm{~nm}$ & Nanoparticles, quantum dots, nanoshells, nanorings \\
Two dimensions $<100 \mathrm{~nm}$ & Nanotubes, fibres, nanowires \\
One dimension $<100 \mathrm{~nm}$ & Thin films, layers and coatings \\
\hline
\end{tabular}

There are two types of nanomaterials:

1. „Non-intentionally-made nanomaterials", which refers to nano-sized particles or materials that belong naturally to the environment (e.g. proteins, viruses, nanoparticles produced during volcanic eruptions, etc.) or that are produced by human activity without intention;

2. „intentionally-made“ nanomaterials, which refers to nanomaterials produced deliberately through a defined fabrication process. [3]

Among variety of their possible applications, novel nanomaterials have been also developed as imaging contrast agents [4-7] and drug delivery systems [8-11], for early detection and treatment of multiple diseases. Figure 1. schematically illustrates the applications of nanotechnology in the diagnosis and therapy of multiple diseases. [12] 


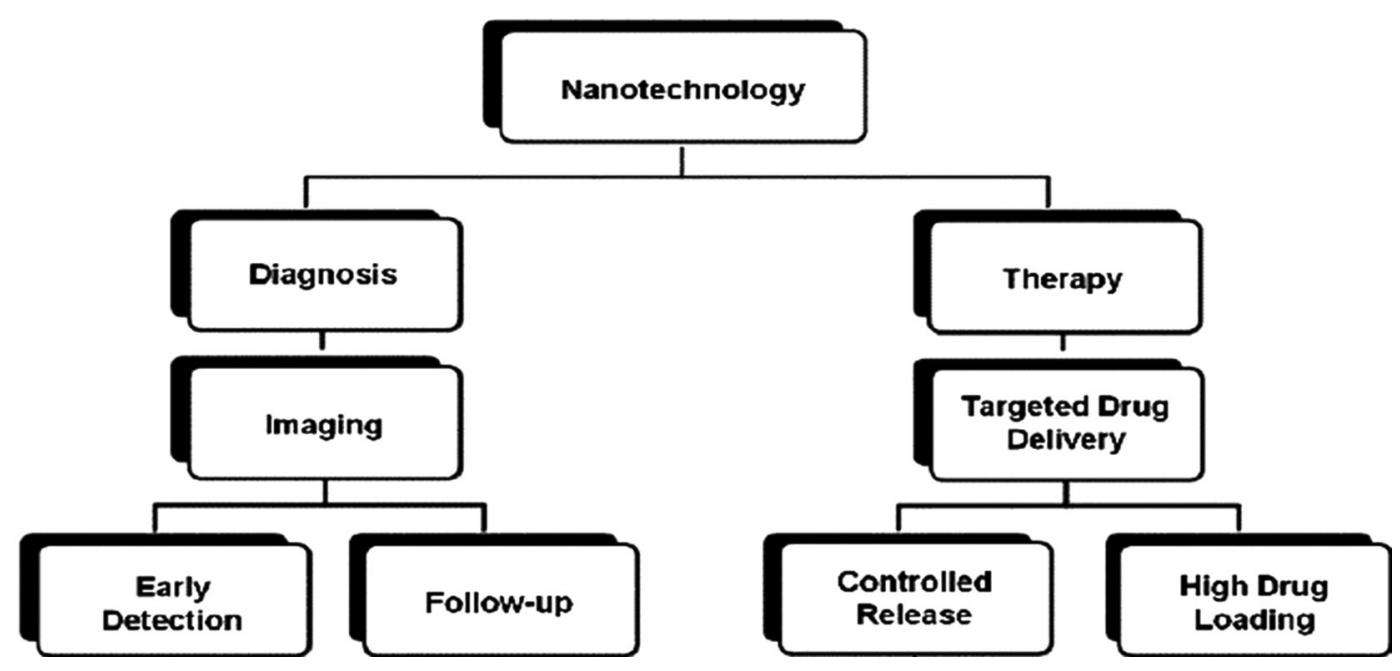

Fig. 1. Scheme of nanotechnology applications in diagnosis and therapy of multiple diseases Modified from Fig. 3. in [12]

Cancer is known to develop via a multistep carcinogenesis process entailing numerous cellular physiological systems such as cell signaling and apoptosis, making it a highly incomprehensible and complex disease. [13] Nanotechnology has brought revolution in the field of oncology, by enabling early detection, accurate diagnosis and treatment approaches that are impossible to achieve using conventional technologies.

Nanodevices made of different materials, in different sizes, shapes and with various chemical and surface properties, have already been engineered for applications in cancer diagnosis and/or therapeutics. Among them, dendrimers and quantum dots are gaining significance as both, diagnostic and therapeutic tools.

\section{DENDRIMERS}

As a new class of polymeric materials, dendrimers are repeatedly branched macromolecules or nano- sized, radially symmetric molecules with welldefined, homogeneous and monodisperse structure consisting of tree-like arms or branches. [14] Hence they were given the corresponding name "dendrimer", that is derived from Greek words "dendri" (tree) and "meros" (part). Over the past two decades since the term "dendrimer" was formally defined, research interest in these molecules has gradually evolved from a primary focus on overcoming purely synthetic challenges to include aesthetic and theoretical perspectives, and, more recently, with the ongoing flurry of "nanobiotechnology" advances, to develop practical and commercial applications for these elegant nanodevices. [15] Basic structure of dendrimer is shown in Figure 2. [16]

It is usually characterized by a central core, from where the chains extend radially; branching points of chains, whose repetition cycles in direction from the centre to the periphery determine generation number of dendrimers; and a highly functional periphery, consisting of end-groups of the chains. End-groups

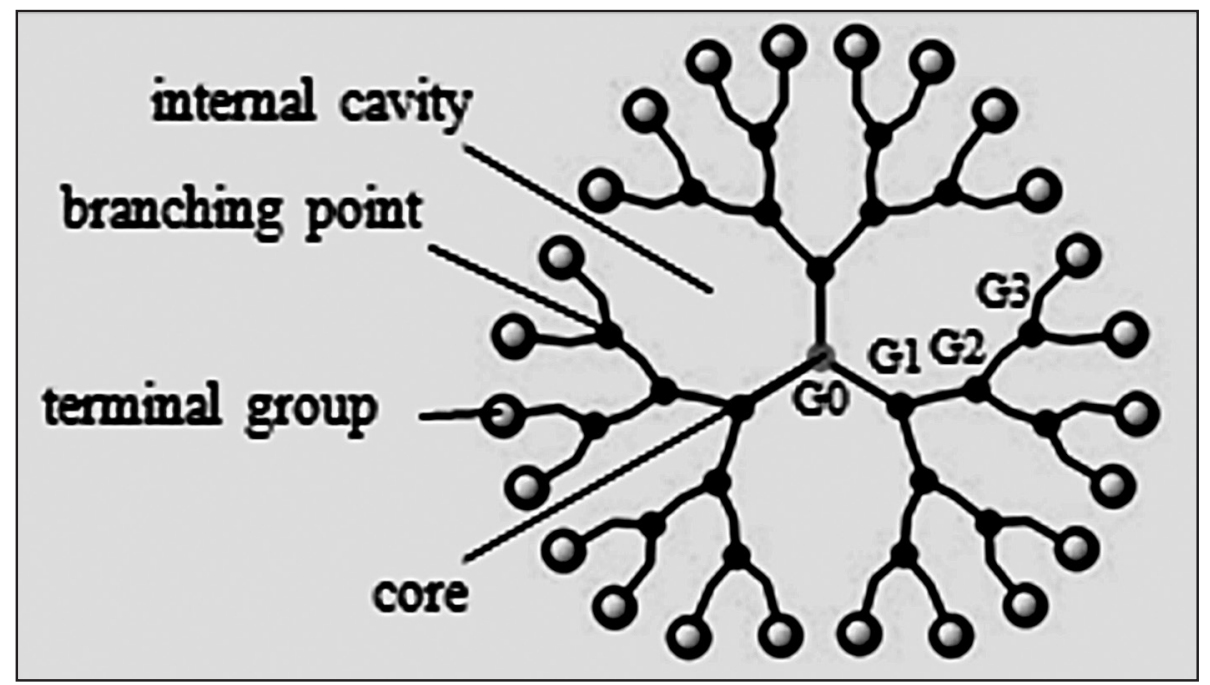

Fig. 2. Basic structure of dendrimer Modified from Fig. 1 in [16] 
are also generally referred to as "terminal groups" or "surface groups" of dendrimers. [17] Cavities, located within the interior structure of the dendrimer, are the result of void spaces between the chains. The generation number of the dendrimer can be easily determined by counting the number of branch points as one proceeds from the core to the periphery [18] and is designated as $\mathrm{G}$, with addition of the corresponding number of generations. The core of the dendrimer is sometimes denoted generation "zero" (G0). Since each branching point creates new generation of two offspring branches, each new generation is more densely packed. However, there continues to be a debate about the exact structure of dendrimers, in particular whether they are fully extended with maximum density at the surface, or whether the end-groups fold back into a densely packed interior. [19]

Dendrimers are synthetically built from a starting atom, such as nitrogen, to which carbon and other elements are added by a repeating series of chemical reactions that produce a spherical branching structure. [20] By repeating this process where successive layers are adjoined, the sphere diameter can be linearly expanded to the required size. The result is a spherical macromolecular structure whose size is similar to small proteins such as hemoglobin and albumin. Due to their dimensional length scaling, narrow size distribution, and other biomimetic properties, dendrimers are often referred to as "artificial proteins". [21] Dendritic volume increases cubically with generation, while dendritic molecular weight increases exponentially. [22] However, their intrinsic viscosity will increase only to a certain generation of the dendrimer, and then begin to decline. The decline in viscosity is a consequence of prohibiting the interaction of the outer branches between molecules at a higher generation. [23] Dendrimers' solubility is strongly influenced by the properties of their surface groups. Dendrimers terminated in hydrophilic groups are soluble in polar solvents, while dendrimers having hydrophobic end groups are soluble in non-polar solvents. [24] Due to the accessibility of the groups by globular shape, the solubility can be controlled. As an example, dendrimers with very hydrophobic interiors such as polyethers and polycarbosilanes can be made water soluble by introducing hydrophilic groups into their surface groups [25] and water soluble dendrimers can be made hydrophobic by converting their surface groups into hydrophobic units. [26] By analogy to electron-saturation levels found in elemental atoms, dendrimers possessing unfilled monomer shells are very reactive at the molecular level via their terminal functional groups, while dendrimer species possessing saturated monomer shells, are not autoreactive, nor do they react with reagents possessing a compatible surface functionality. [27] Dendritic species possessing an unsaturated outer monomer shell of ester and amine domains exhibit autoreactive behavior. [28]

\subsection{Synthesis}

There are two main synthesis approaches for the construction of dendrimers, divergent and convergent aproaches. The early synthetic efforts in dendrimer synthesis applied the divergent synthesis procedure. [29] In the divergent approach, the construction of the dendrimer takes place in a stepwise manner, starting from the core and building up the molecule towards the periphery using two basic operations: coupling of the monomer and deprotection or transformation of the monomer end-group to create a new reactive surface functionality and then coupling of a new monomer. [16] These operations can be repeated multiple times to form different generations of dendrimers. A schematic representation of divergent growth method is shown in Figure 3. [30]

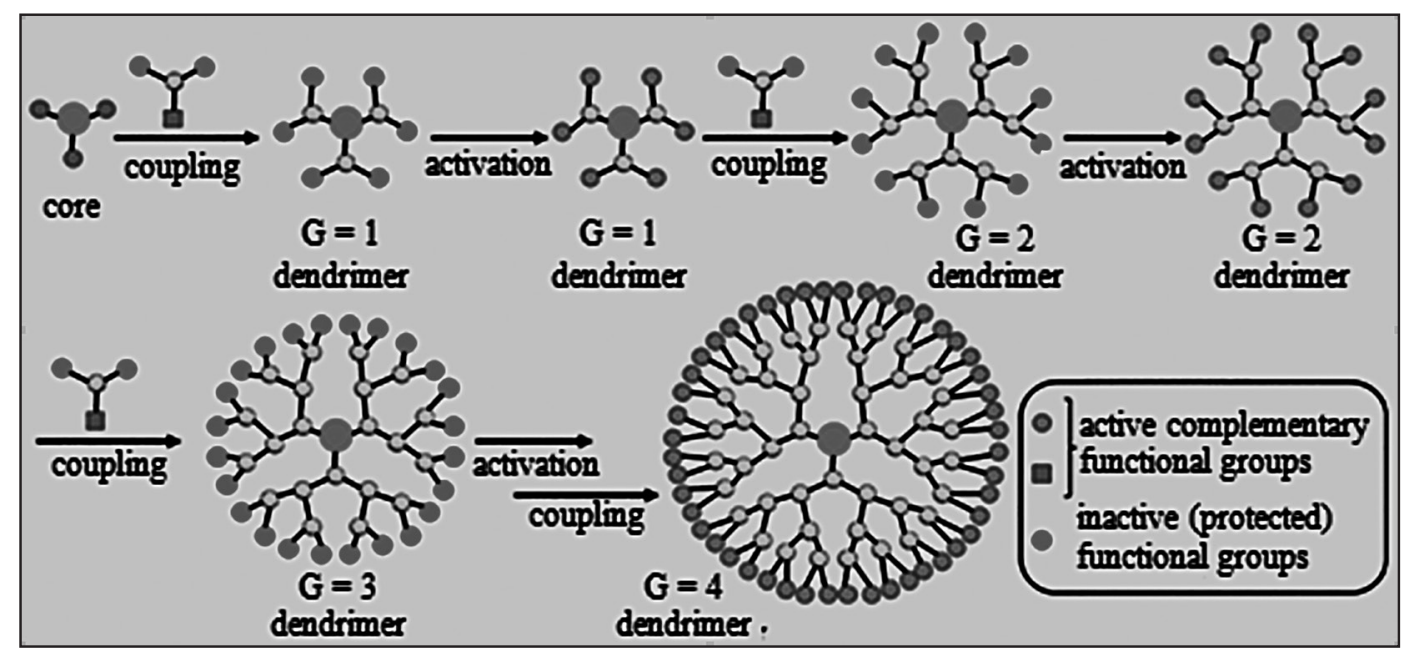

Fig. 3. Divergent growth method [30]

Although the divergent method is able to construct highgeneration dendrimers [31], the major disadvantage of this approach is that the incomplete growth and the side reactions lead to imperfect dendrimers. [32] Moreover, purification and separation of structurally perfect dendrimers from defective dendrimers by standard purification techniques are very difficult to achieve because the compounds are very similar, both chemically and in size. [33] 
Convergent approach of dendrimer synthesis emerged as alternative to overcome the issues of purity and structural defects, associated with divergent synthesis. Convergent synthesis is applied in the synthesis of complex molecules and involves fragment coupling and independent synthesis. [22] Similarly to the divergent approach, the dendrimer is constructed stepwise, however, starting from the end groups and progressing inwards, creating branched polymeric arms, called dendrons. [24] When dendrons are large enough, in the final step they are linked together to a core molecule for getting complete dendrimer structure. [34] A schematic representation of convergent growth method is shown in Figure 4. [30]

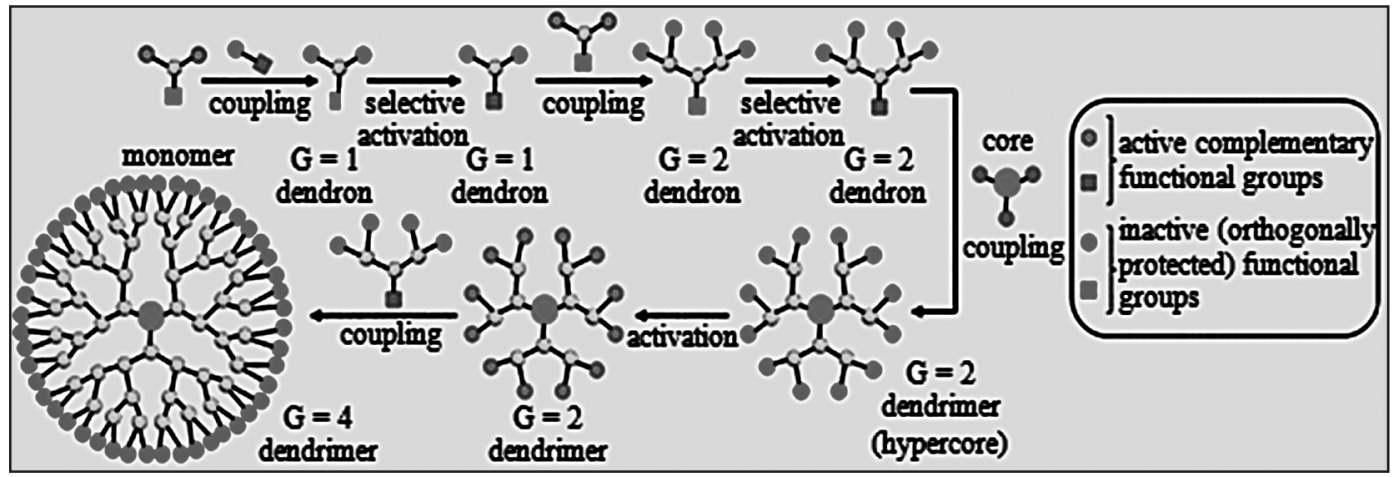

Fig. 4. Convergent growth method [30]

Key advantages of convergent growth method over divergant are minimization the occurrence of defects in the final structure and easier purification process, which give higher purity of the dendrimers. The main disadvantage is that convergent approach does not allow the formation of high generations because steric problems occur in the reactions of the dendrons and the core molecule. Generaly lower yields obtained using the convergent procedure are the possible reason why this approach is generally used for laboratory scale dendrimer synthesis. For commercial scale production, divergent synthesis is still favored. [35]

\subsection{Application}

Pharmacokinetic properties are one of the most significant aspects that need to be considered for the successful biomedical application of dendrimers, for instance, targeted drug delivery, imaging, photodynamic therapy, and neutron capture therapy. [18] Various dendrimers which should be devoid of toxicity and immunogenicity can be important in cancer research, diagnosis and treatment. Dendrimeric structures like glycopeptide or peptide dendrimers have been intensively studied for their use as diagnostic or therapeutic tools and it is shown that this dendrimeric structures can display direct toxicity against tumor cells. They also possess a great potential that can be utilized in antitumor immunotherapy, either as vaccines or as adjuvants. Various dendrimers can serve as carriers for active toxic compounds or vectors for anti/tumor gene therapy thus increasing the therapeutic efficacy and specificity, and simultaneously minimizing adverse effects which limit current therapeutic modalities. [36]

Scientist in their studies discussed about application of dendrimer-drug conjugates by oral, intravenous, intraperitoneal, intratumoral, transdermal, ocular, etc. and they are focused on their use as unimolecular micelles and "dendritic boxes" for the noncovalent encapsulation of drug molecules. [37,38] Initial studies show that drugs are delivered by dendrimers by electrostatic interaction, simple encapsulation, or covalent conjugation. [37] Using dendritic unimolecular micelles rather than conventional polymeric micelles is an advantage because the micellar structure is maintained at all concentrations because the hydrophobic segments are covalently connected. Example of this is study in which DNA was complexed with PAMAM dendrimers for gene delivery applications, and hydrophobic drugs and dye molecules were incorporated into various dendrimer cores. [38]

In preclinical studies, dendrimers have been tested as contrast agents for magnetic resonance. A new class of magnetic resonance imaging contrast agents, Gd(III)-DTPA -based PAMAM dendrimers, with large proton relaxation enhancements and high molecular relaxivities were developed by Wiener et al. These 6th generation PAMAM dendrimers possess 192 reactive terminal amines, which can be conjugated to the chelating ligand 2-(4-isothiocyanato-benzyl)-6-methyl(DTPA) through a thiourea linkage and this dendrimer has a relaxivity a six times higher than that of free Gd(III)-DTPA complex. Experimental studies on rabbits show excellent MRI images of blood vessels and long blood circulation times ( $>100 \mathrm{~min}$ ) upon intravenous injection. The results of study, in which dendrimerbased MRI contrast agents were synthesized and the relationship between relaxivity and dendrimer generations using Gd(III)-DTPA based PAMAM dendrimers were investigated, revealed that relaxivities increased as the dendrimer generation increased, but there was no significant increase in relaxivities beyond 7th generation. [38]

Dendrimers offer several mechanisms of interactions with different classes of drugs. The different types of drug-dendrimer interactions proposed for drug delivery are presented in Figure 5. Broadly these interactions can be categorized under two categories viz. physical and chemical bonding. [39] 


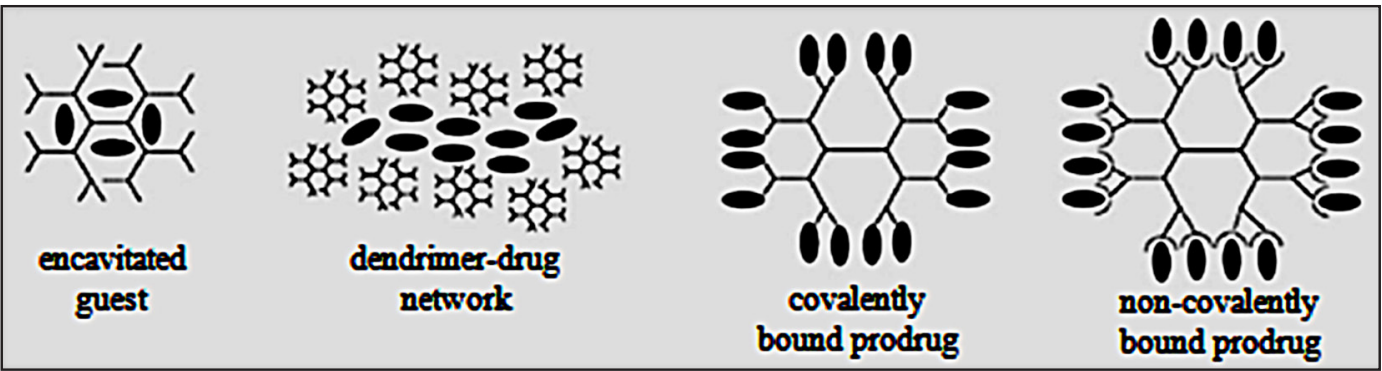

Fig. 5. Different types of drug-dendrimer interactions [39]

\section{QUANTUM DOTS}

The quantum dot (QD) is defined as an artificially structured system with the capacity to load electrons. Its special physicochemical properties differentiate it from other naturally occurring biogenic and anthropogenic nanoparticles. [40] QDs are one of the most promising nanocrystals with unique optical and chemical properties. They are semiconductor nanocrystals that range from $2 \mathrm{~nm}$ to $10 \mathrm{~nm}$ in diameter and consist of elements from groups II to VI or III to V. In Figure 6. [41] is shown quantum dots which consist a semiconductor core, over coated by a shell (e.g. ZnS) to improve optical properties, and a cap enabling improved solubility in aqueous buffers. QDs have a number of beneficial characteristics for spectroscopy, such as high fluorescence intensity, long lifetime, and good resistance to photo bleaching and great advantages over traditional organic fluorescent dyes. [41]

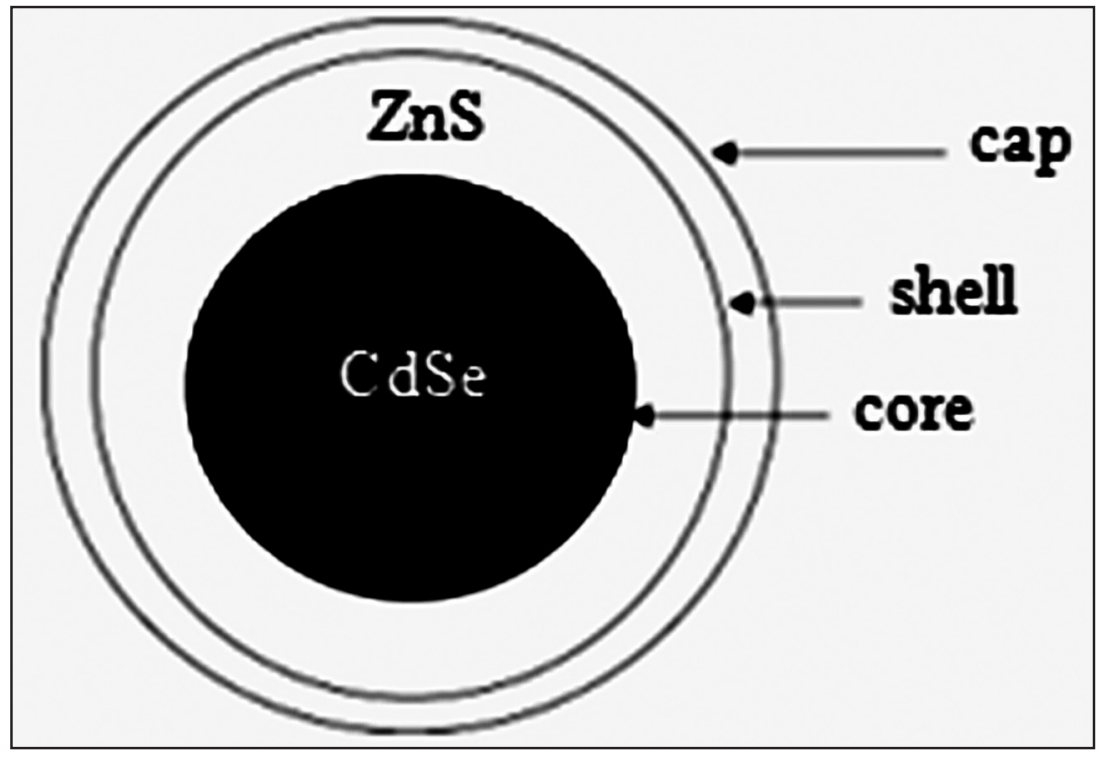

Fig. 6. Schematic representation of a quantum dot [41]

\subsection{Synthesis}

Figure 7. shows colloidal synthesis of CdSe quantum dots. [42] Several routes can be used to synthesize QDs, like top-down processing methods and bottomup approach. Molecular beam epitaxy (MBE), ion implantation, e-beam lithography, and X-ray lithography are included in top-down processing methods. Colloidal QDs are prepared by self-assembling the solution following a chemical reduction using the alternative bottom-up approach. For making the QDs, in the approaches of top-down, a bulk semiconductor is thinned. Reactive-ion etching, electron beam lithography and/or wet chemical etching are commonly used for the achieve QDs of diameter approximately $30 \mathrm{~nm}$. The desired packing geometries achievable the systematic experiments on quantum confinement effect, controlled shapes and sizes. To fabricate arrays of zero-dimension dots, also focused ion or laser beams have been used and major disadvantages with these processes are incorporation of impurities into the QDs and structural imperfections. [43] 


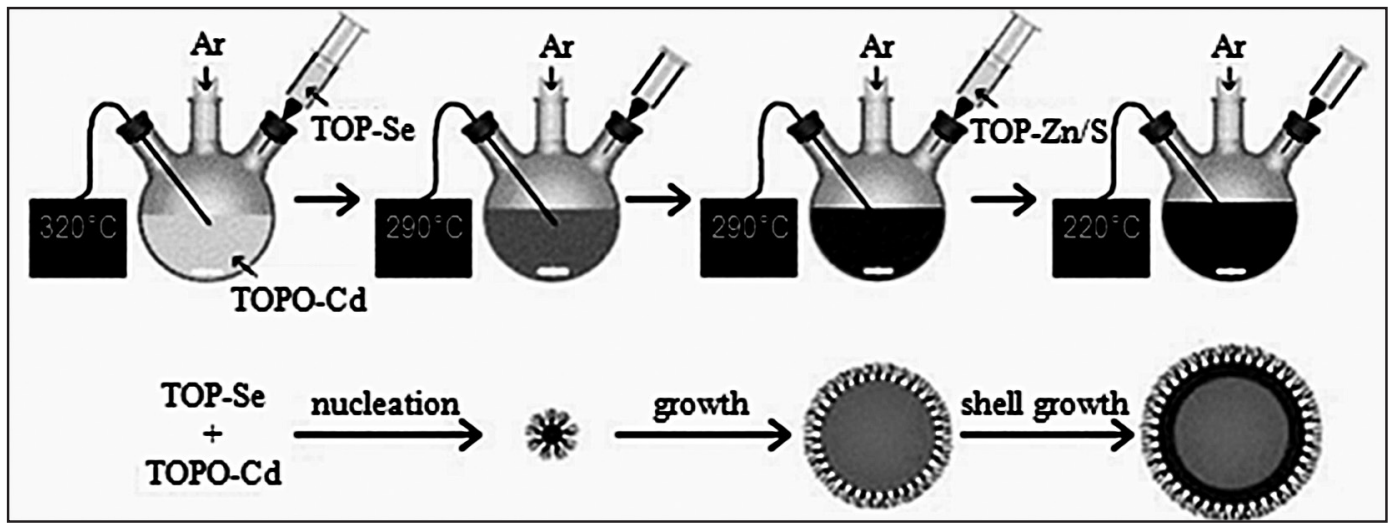

Fig. 7. Colloidal synthesis of CdSe quantum dots [43]

\subsection{Application}

High sensitivity for simultaneous cancer molecular imaging and targeted therapy is afforded by the brightness of QD-based multifunctional probes. The sensitivity of QD-based molecular imaging can be two to three orders larger than that of routine fluorescent dyes, for spectrum application. [44] NIRQDs are suitable for in vivo imaging with high signal to back ground ratio and they can be detected in deep tissues. Magnetic resonance spectroscopy (MRS), optical fluorescence imaging, single-photon-emission computed tomography (SPECT), molecular MRI, optical bioluminescence imaging, targeted ultrasound, and positron emission tomography (PET) are included in molecular imaging modalities. Because of the potential to detect early stage cancers and their metastases active targeting of cancer antigens (molecular imaging), in case of imaging probes, has become an area of tremendous interest. In Table 2 are summarized major recent developments in this regard. [45]

Table 2. Application of QDs in molecular, cell, tissue, animal model cancer imaging [45]

\begin{tabular}{|c|c|c|c|}
\hline QDs used & Major findings & Advantages & Problems \\
\hline \multicolumn{4}{|l|}{ Molecular imaging } \\
\hline $\begin{array}{l}\text { QD-HER2 (525 nm), QD-ER } \\
\text { (565 nm), QD-PR }(605 \mathrm{~nm}) \text {, } \\
\text { QD-EGFR }(655 \mathrm{~nm}), \\
\text { QD-mTOR }(705 \mathrm{~nm})\end{array}$ & $\begin{array}{l}\text { QDs can be used for } \\
\text { multiplexed and quantitative } \\
\text { detection of tumor } \\
\text { biomarkers }\end{array}$ & $\begin{array}{l}\text { High sensitivity; Increased } \\
\text { resolution; Decreased } \\
\text { autofluorescence }\end{array}$ & $\begin{array}{l}\text { Better conjugation chemistry } \\
\text { for well-controlled bio- } \\
\text { ligand are needed }\end{array}$ \\
\hline RGD-conjugated QDs & $\begin{array}{l}\text { QDs readily reveal } \\
\text { the angiogenic tumor } \\
\text { vasculature, with the highest } \\
\text { angiogenic activity occurring } \\
\text { in the periphery of the tumor }\end{array}$ & & $\begin{array}{l}\text { The number of bio-ligands } \\
\text { per nanoparticle needs to be } \\
\text { characterized }\end{array}$ \\
\hline \multicolumn{4}{|l|}{ Cancer cell imaging } \\
\hline Trastuzumab-QDs (800 nm) & $\begin{array}{l}\text { Single breast cancer cell can } \\
\text { be observed with QDs }\end{array}$ & $\begin{array}{l}\text { High brightness; Resistance } \\
\text { to photobleachings }\end{array}$ & $\begin{array}{l}\text { The methods current used } \\
\text { for delivery into cells are not } \\
\text { highly efficient }\end{array}$ \\
\hline Bio-conjugated QDs & $\begin{array}{l}\text { Stem cells can be effectively } \\
\text { labeled by QDs during both } \\
\text { proliferation and multilin- } \\
\text { eage differentiation for long } \\
\text { term }\end{array}$ & InP/ZnS QDs are nontoxic & $\begin{array}{l}\text { It is difficult to prepare InP/ } \\
\text { ZnS QDs because of the } \\
\text { sensitivity of precursors } \\
\text { and surfactants toward the } \\
\text { reaction environment in } \\
\text { obtaining good InP QDs }\end{array}$ \\
\hline Bio-conjugated InP/ZnS QDs & $\begin{array}{l}\text { InP/ZnS QDs can be used as } \\
\text { non-cadmium-based safe } \\
\text { and efficient optical imaging } \\
\text { nanoprobes }\end{array}$ & - & \\
\hline
\end{tabular}




\begin{tabular}{|l|l|l|l|}
\hline \multicolumn{1}{|c|}{ QDs used } & \multicolumn{1}{|c|}{ Major findings } & \multicolumn{1}{|c|}{ Pdvantages } \\
\hline Cancer tissue imaging & $\begin{array}{l}\text { The expression of Her2 of } \\
\text { human breast cancer tissue } \\
\text { was detected }\end{array}$ & $\begin{array}{l}\text { Highly efficient, nontoxic, } \\
\text { quantitative, sensitive, } \\
\text { convenient }\end{array}$ & $\begin{array}{l}\text { Data from clinical trials } \\
\text { about QDs comparing with } \\
\text { "Gold standard" is required. }\end{array}$ \\
\hline AnR2-QDs & $\begin{array}{l}\text { For the first time, authors } \\
\text { have demonstrated the } \\
\text { ability to directly follow } \\
\text { the specific binding } \\
\text { of nanoparticles to } \\
\text { biomolecules expressed } \\
\text { on tumor neovascular } \\
\text { endothelium in mouse model }\end{array}$ & $\begin{array}{l}\text { Portends the promise } \\
\text { of studying nanoscale } \\
\text { structures interacting with } \\
\text { microscale entities in living } \\
\text { subjects at the cellular-to- } \\
\text { subcellular level }\end{array}$ & $\begin{array}{l}\text { QDs in animal model are still } \\
\text { controversial limiting the } \\
\text { clingl use of QDs }\end{array}$ \\
\hline NIR QDs (840 nm) & $\begin{array}{l}\text { Bowel lymphatics are a key } \\
\text { determinant of peritoneal } \\
\text { lymph flow, because bowel } \\
\text { resection shifts lymph flow } \\
\text { directly to the intrathoracic } \\
\text { lymph nodes via chest wall } \\
\text { lymphatics }\end{array}$ & $\begin{array}{l}\text { QDs can be excellently } \\
\text { visualized in vivo using IVM }\end{array}$ & - \\
\hline
\end{tabular}

Figure 8. shows a possible schematic of the QD bioconjugate for the detection of tumor cell biomarkers. [46]

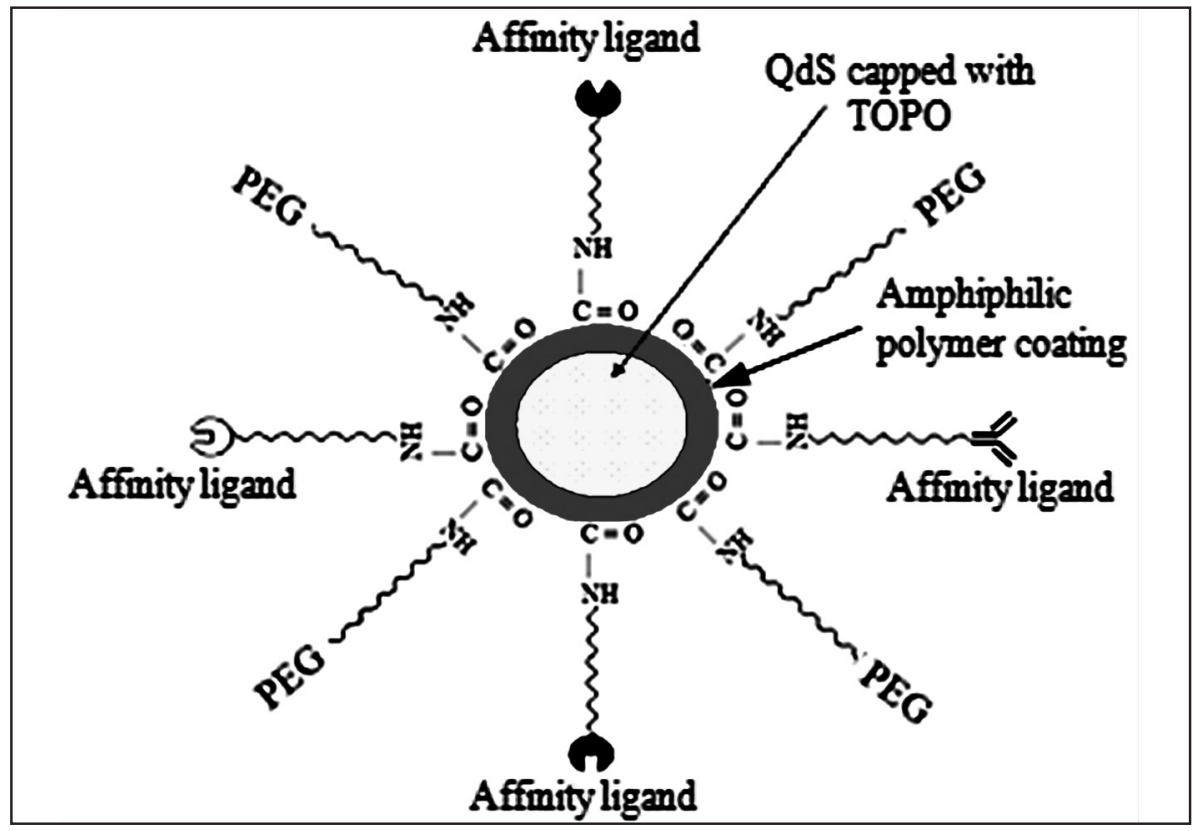

Fig. 8. Multifunctional QDs usually employed for targeting tumor cells [46]

With various affinity ligands (antibody, peptide, drug, inhibitor, etc.) specific for the tumor cell biomarkers are conjugated QDs. A comparative study of ability of conventional immunohistochemistry (IHC) and quantum dots immunofluorescence histochemistry (QDs-IHC) for the detection of caveolin-1 and PCNA in the lung cancer tissue microarray concluded that both methods could precisely detect the expression of caveolin-1 and PCNA markers. In this study is shown that with QDs-IHC is obtained a higher sensitivity. [47] An important function in cancer progression has human epidermal growth factor receptor 2 (HER2) and it is overexpressed in approximately $25 \%$ to $30 \%$ $\mathrm{BC}$ patients. The advantages of QD-based IHC have been well documented since Wu et al. labeled HER2 on human BC cells (SK-BR-3) and mouse mammary tumor sections by QD-IgG conjugates for the first time. This was cheaper, easier, less time-consuming, and could relieve the medical burden, especially for developing countries. Various studies have reported the successful detection for BC by QD-HER2 conjugates. [48] It is clear that as biocompatible QDs are developed they will make powerful basic probes and research tools, and the delivery of QDs/QD biocomjugates is strongly affected by the nature of both the QDs conjugates and the cell types utilized. A lot of techniques about QDs have been improved with the development of new QDs, major issues need to be resolved in the near future. [45] 


\section{CONCLUSION}

The dendrimers and the quantum dots are of great importance in the diagnosis and treatment of malignant diseases in the future. The advantages of applying the

\section{REFERENCES}

1. Taniguchi N. (1974). On the basic concept of nanotechnology, In Proceedings of the International Congress on Production Engineering, Tokyo, 18-23.

2. Kalyane S. Basic of Nano Technology. Horizon Books, 2017. pp. 8.

3. NANOTECHNOLOGIES: Principles, Applications, Implications and Hands-on Activities - A compendium for educators, pp. 20. More information is available at: https://ec.europa.eu/research/industrial_technologies/ pdf/nano-hands-on-activities_en.pdf

4. Reguera J., Jiménez de Aberasturi D., Henriksen-Lacey M., Langer J., Espinosa A., Szczupak B., Wilhelm C., LizMarzán L.M. Janus plasmonic-magnetic gold-iron oxide nanoparticles as contrast agents for multimodal imaging. Nanoscale 2017; 9(27): 9467-9480.

5. Liopo A., Su R., Oraevsky A.A. Melanin nanoparticles as a novel contrast agent for optoacoustic tomography. Photoacoustics 2015; 3(1): 35-43.

6. Cabrera-García A., Vidal-Moya A., Bernabeu A., PachecoTorres J., Checa-Chavarria E., Fernández E., Botella P. GdSi Oxide Nanoparticles as Contrast Agents in Magnetic Resonance Imaging. Nanomaterials 2016; 6(6), 109.

7. Chen F., Ma M., Wang J., Wang F., Chern S.X. Zhao E.R., Jhunjhunwala A., Darmadi S., Chen H., Jokerst J.V. Exosome-like silica nanoparticles: a novel ultrasound contrast agent for stem cell imaging. Nanoscale 2017; 9(1): 402-411.

8. Stergar J., Ban I., Gradišnik L., Maver U. Novel drug delivery system based on $\mathrm{NiCu}$ nanoparticles for targeting various cells. Journal of Sol-Gel Science and Technology 2017; 1-9.

9. Shrivastav A., Kim H.Y., Kim Y.R. Advances in the Applications of Polyhydroxyalkanoate Nanoparticles for Novel Drug Delivery System. BioMed Research International 2013; 2013: 12 pages.

10. Yu X., Trase I., Ren M., Duval K., Guo X., Chen Z. Design of nanoparticle-based carriers for targeted drug delivery. Journal of Nanomaterials 2016; 2016: 15 pages.

11. Lv Y., Hao L., Hu W., Ran Y., Bai Y., Zhang L. Novel multifunctional $\mathrm{pH}$-sensitive nanoparticles loaded into microbubbles as drug delivery vehicles for enhanced tumor targeting. Scientific Reports 2016; 6: 9 pages.

12. Amiri H., Saeidi K., Borhani P., Manafirad A., Ghavami M., Zerbi V. Alzheimer's Disease: Pathophysiology and Applications of Magnetic Nanoparticles as MRI Theranostic Agents, ACS Chemical Neuroscience 2013; 4(11): 1417-1429. nanostructures have already been confirmed through a number of studies around the world, while a large number of them are carried out and gives promising results. This will certainly help in early diagnosis and quality of this type of disease and of course conditional on a better treatment outcome.

13. Dhiman V., Hari Kumar S. L. Nanotechnology in cancer therapy. Journal of Drug Delivery \& Therapeutics 2013; 3(5): 137-142.

14. Hari B.N.V., Kalaimagal K., Porkodi R., Gajula P.K., Ajay J.Y. Dendrimer: Globular Nanostructured Materials for Drug Delivery, International Journal of PharmTech Research 2012; 4(1): 432-451.

15. Srinivasa-Gopalan S., Yarema K. J. „Dendrimers in Cancer Treatment and Diagnosis" in book: Nanotechnologies for the Life Sciences. Wiley, 2007, pp. 1.

16. Zhao F.G., Li W.S. Dendrimer/inorganic nanomaterial composites: Tailoring preparation, properties, functions, and applications of inorganic nanomaterials with dendritic architectures. Science China Chemistry 2011; 54(2): 286-301.

17. Boas U, Christensen J.B., Heegaard P.M.H. Dendrimers: design, synthesis and chemical properties. Journal of Materials Chemistry 2006; 16: 3785-3798.

18. Massoudi A., Vahedi H., Louie O., Sajjadiffar S. Theoretical Determination of Molecular Weight of AB2 Dendrimers. E-Journal of Chemistry 2009; 6(3): 681-684.

19. Abbasi E., Aval S.F., Akbarzadeh A., Milani M., Nasrabadi H.T., Joo S.W., Hanifehpour Y., Nejati-Koshki K., PashaeiAsl R. Dendrimers: synthesis, applications, and properties. Nanoscale Research Letters 2014; 9(1): 247.

20. Roseita E., Tomalia D.A. Poly (amidoamine) (PAMAM) dendrimers: from biomimicry to drug delivery and biomedical applications. Drug Delivery Today 2001; 6: 427-436.

21. 21. Svenson S., Tomalia D.A. Dendrimer in biomedical applications - reflection on the field. Advanced Drug Delivery Reviews 2005; 57: 2106-2129.

22. Singh U., Dar M.M., Hashmi A.A. Dendrimers: synthetic strategies, properties and applications. Oriental Journal of Chemistry 2014; 30(3): 911-922.

23. Shinde G.V., Bangale G.S., Umalkar D.G., Rathinaraj B.S., Yadav C.S., Yadav P. Dendrimers. Journal of pharmaceutical and biomedical sciences 2010; 3(3): 1-8.

24. Klajnert B., Bryszewska M. Dendrimers: properties and applications. Acta Biochimica Polonica 2001; 48(1): 199-208.

25. Inoue K. Functional dendrimers, hyperbranched and star polymers. Progress in Polymer Science 2000; 25: 453571.

26. Moorefield C.N., Newkome G.R. In Advances in dendritic macromolecules, Newkome GR (Ed) vol. 1, Greenwich, CT: JAI Press, 1994, p. 1. 
27. Tomalia D.A. Birth of a New Macromolecular Architecture: Dendrimers as Quantized Building Blocks for Nanoscale Synthetic Organic Chemistry. Aldrichimica Acta 2004; 37(2): 39-57.

28. Tomalia D.A. The dendritic state. Materials Today 2005; 8(3): 34-46.

29. Pittelkow M., Christensen J.B. Convergent Synthesis of Internally Branched PAMAM Dendrimers. Organic Letters 2005; 7(7): 1295-1298.

30. Sowinska M., Urbanczyk-Lipkowska Z. Advances in the chemistry of dendrimers. New Journal of Chemistry 2014; 38(6), 2168-2203.

31. Cheng Y., Xu Z., Ma M., Xu T. Dendrimers as Drug Carriers: Applications in Different Routes of Drug Administration. Journal of pharmaceutical sciences 2008; 97(1): 123143.

32. Rai A.K., Tiwari R., Maurya P., Yadav P. Dendrimers: a potential carrier for targeted drug delivery system. Pharmaceutical and Biological Evaluations 2016; 3(3): 275-287.

33. Sowinska M., Urbanczyk-Lipkowska Z. Advances in the chemistry of dendrimers. New Journal of Chemistry 2014; 38(6), 2168-2203.

34. Gupta V., Nayak S.K. Dendrimers: a Review on Synthetic Approaches. Journal of Applied Pharmaceutical Science 2015; 5(03): 117-122.

35. Dhote V., Dhote K., Mishra D.K., Mishra S. Dendrimer: novel strategies for drug delivery system. Asian Journal of Pharmaceutical Education and Research 2015; 4(4): 1-18.

36. Šebestík J., Reiniš M., Ježek, J. „Cancer“ in Biomedical Applications of Peptide-, Glyco- and Glycopeptide Dendrimers, and Analogous Dendrimeric Structures. Springer, 2012, pp 175-182.

37. Šebestík J., Reiniš M., Ježek, J. „Dendrimers in Drug Delivery" in book Biomedical Applications of Peptide-, Glyco- and Glycopeptide Dendrimers, and Analogous Dendrimeric Structures. Springer, 2012, pp 131-140.

38. Noriega-Luna B., Godínez L. A., Rodríguez F. J., Rodríguez A., Zaldívar-Lelo de Larrea G., Sosa-Ferreyra C. F.,
Mercado-Curiel R.F., Manríquez J., Bustos E. Applications of Dendrimers in Drug Delivery Agents, Diagnosis, Therapy, and Detection. Journal of Nanomaterials 2014; 2014: 19 pages.

39. Madaan K., Kumar S., Poonia N., Lather V., Pandita D. Dendrimers in drug delivery and targeting: Drugdendrimer interactions and toxicity issues. Journal of Pharmacy and Bioallied Sciences 2014; 6(3): 139-150.

40. Liu L., Miao Q., Liang, G. Quantum Dots as Multifunctional Materials for Tumor Imaging and Therapy. Materials 2013; 6(2): 483-499.

41. Ghasemi Y., Peymani P., Afifi S. Quantum dot: magic nanoparticle for imaging, detection and targeting. Acta Biomedica 2009; 80: 156-165.

42. Valizadeh A., Mikaeili H., Samiei M., Farkhani S.M., Zarghami N., Kouhi M., Akbarzadeh A., Davaran S. Quantum dots: synthesis, bioapplications, and toxicity. Nanoscale Research Letters 2012; 7(1): 480.

43. Kango S., Kalia S., Thakur P., Kumari B., Pathania D. Semiconductor-Polymer Hybrid Materials. Advances in Polymer Science 2015; 267: 283-312.

44. Min F., Chun-wei P., Dai-Wen P., Yan L. Quantum Dots for Cancer Research: Current Status, Remaining Issues, and Future Perspectives. Cancer Biol Med. 2012; 9(3): 151163.

45. Peng C.W., Li Y. Application of Quantum Dots-Based Biotechnology in Cancer Diagnosis: Current Status and Future Perspectives. Journal of Nanomaterials 2010; 2010: 11 pages.

46. Washist S.K., Tewari R., Bajpai R.P., Bharadwaj L.M., Raiteri R. Review of Quantum Dot Technologies for Cancer Detection and Treatment. AZojono Journal of Nanotechnology Online 2006; 2: 1-14.

47. Malik P., Gulia S., Kakkar R. Quantum dots for diagnosis of cancers. Advanced Materials Letters 2013; 4(11): 811822.

48. Xiao Y., Gao X., Gannot G., Emmert-Buck M.R., Srivastava S., Wagner P.D., Amos M.D., Barker P.E. Quantitation of HER2 and telomerase biomarkers in solid tumors with IgY antibodies and nanocrystal detection, International Journal of Cancer 2008; 122: 2178-2186. 